\title{
Guidelines for the management of biliary tract and ampullary carcinomas: surgical treatment
}

\author{
Satoshi Kondo ${ }^{1}$, Tadahiro Takada ${ }^{2}$, Masaru Miyazaki ${ }^{3}$, Shuichi Miyakawa ${ }^{4}$, Kazuhiro Tsukada ${ }^{5}$, \\ Masato Nagino ${ }^{6}$, Juni Furuse ${ }^{7}$, Hiroya Saito ${ }^{8}$, Toshio Tsuyuguchi ${ }^{9}$, Masakazu Yamamoto ${ }^{10}$, \\ Masato Kayahara $^{11}$, Fumio Kimura ${ }^{3}$, Hideyuki Yoshitomi ${ }^{3}$, Satoshi Nozawa ${ }^{3}$, Masahiro Yoshida ${ }^{2}$, \\ Keita Wada ${ }^{2}$, SATOSHI Hirano ${ }^{1}$, Hodaka Amano ${ }^{2}$, and Fumihiko Miura ${ }^{2}$ \\ ${ }^{1}$ Department of Surgical Oncology, Hokkaido University Graduate School of Medicine, N15 W7, Kita-ku, Sapporo 060-8638, Japan \\ ${ }^{2}$ Department of Surgery, Teikyo University School of Medicine, Tokyo, Japan \\ ${ }^{3}$ Department of General Surgery, Chiba University Graduate School of Medicine, Chiba, Japan \\ ${ }^{4}$ Department of Gastroenterological Surgery, Fujita Health University, Toyoake, Japan \\ ${ }^{5}$ Department of Surgery and Science, Graduate School of Medicine and Pharmaceutical Science for Research, University of Toyama, \\ Toyama, Japan \\ ${ }^{6}$ Division of Surgical Oncology, Department of Surgery, Nagoya University Graduate School of Medicine, Nagoya, Japan \\ ${ }^{7}$ Hepatobiliary and Pancreatic Oncology Division, National Cancer Center Hospital East, Chiba, Japan \\ ${ }^{8}$ Department of Radiology, Asahikawa Kosei General Hospital, Asahikawa, Japan \\ ${ }^{9}$ Department of Medicine and Clinical Oncology, Chiba University Graduate School of Medicine, Chiba, Japan \\ ${ }^{10}$ Department of Surgery, Institute of Gastroenterology, Tokyo Women's Medical University, Tokyo, Japan \\ ${ }^{11}$ Department of Gastroenterologic Surgery, Kanazawa University, Graduate School of Medical Science, Kanazawa, Japan
}

\begin{abstract}
The only curative treatment in biliary tract cancer is surgical treatment. Therefore, the suitability of curative resection should be investigated in the first place. In the presence of metastasis to the liver, lung, peritoneum, or distant lymph nodes, curative resection is not suitable. No definite consensus has been reached on local extension factors and curability. Measures of hepatic functional reserve in the jaundiced liver include future liver remnant volume and the indocyanine green (ICG) clearance test. Preoperative portal vein embolization may be considered in patients in whom right hepatectomy or more, or hepatectomy with a resection rate exceeding $50 \%-60 \%$ is planned. Postoperative complications and surgery-related mortality may be reduced with the use of portal vein embolization. Although hepatectomy and/or pancreaticoduodenectomy are preferable for the curative resection of bile duct cancer, extrahepatic bile duct resection alone is also considered in patients for whom it is judged that curative resection would be achieved after a strict diagnosis of its local extension. Also, combined caudate lobe resection is recommended for hilar cholangiocarcinoma. Because the prognosis of patients treated with combined portal vein resection is significantly better than that of unresected patients, combined portal vein resection may be carried out. Prognostic factors after resection for bile duct cancer include positive surgical margins, especially in the ductal stump; lymph node metastasis; perineural invasion; and combined vascular resection due to portal vein and/or hepatic artery invasion. For patients with suspected gallbladder cancer, laparoscopic cholecystectomy is not recommended, and open cholecystectomy should be performed as a rule. When gallbladder cancer
\end{abstract}

Offprint requests to: S. Kondo

Received: October 1, 2007 / Accepted: October 22, 2007 invading the subserosal layer or deeper has been detected after simple cholecystectomy, additional resection should be considered. Prognostic factors after resection for gallbladder cancer include the depth of mural invasion; lymph node metastasis; extramural extension, especially into the hepatoduodenal ligament; perineural invasion; and the degree of curability. Pancreaticoduodenectomy is indicated for ampullary carcinoma, and limited operation is also indicated for carcinoma in adenoma. The prognostic factors after resection for ampullary carcinoma include lymph node metastasis, pancreatic invasion, and perineural invasion.

Key words Biliary tract neoplasms · Bile duct neoplasms · Gallbladder neoplasms · Ampulla of Vater · Surgery · Guidelines

\section{Introduction}

At present, there are no opinions against the suitability of surgical resection as the only curative treatment for biliary tract cancer. Therefore, the first thing to do is to consider the suitability of surgical resection in each patient in whom a diagnosis of biliary tract cancer has been made. Shown in Fig. 1 are postoperative long-term results reported in the Biliary Tract Cancer Statistics Registry in Japan 1988-1997 ${ }^{1}$ for resected patients.

The classification of biliary tract cancers is made according to the Japanese Society of Biliary Surgery Classification of biliary tract carcinoma. ${ }^{2}$ On the basis of the site of occurrence, biliary tract carcinomas are classified as intrahepatic and extrahepatic bile duct cancers, ampullary cancer, and gallbladder cancer. For the classification of hilar cholangiocarcinoma, not only the 


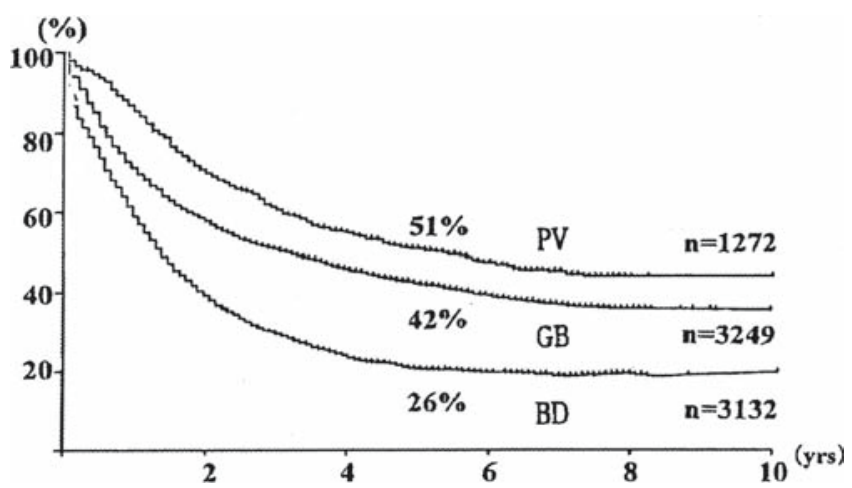

Fig. 1. Survival of patients with resection of gallbladder cancer, bile duct cancer, and cancer of the papilla of Vater. Percentages show 5-year survival rates. ${ }^{1} G B$, Cancer of the gallbladder, $B D$, cancer of the bile duct; $P V$, cancer of the papilla of Vater. Postoperative survival after resection of biliary cancer

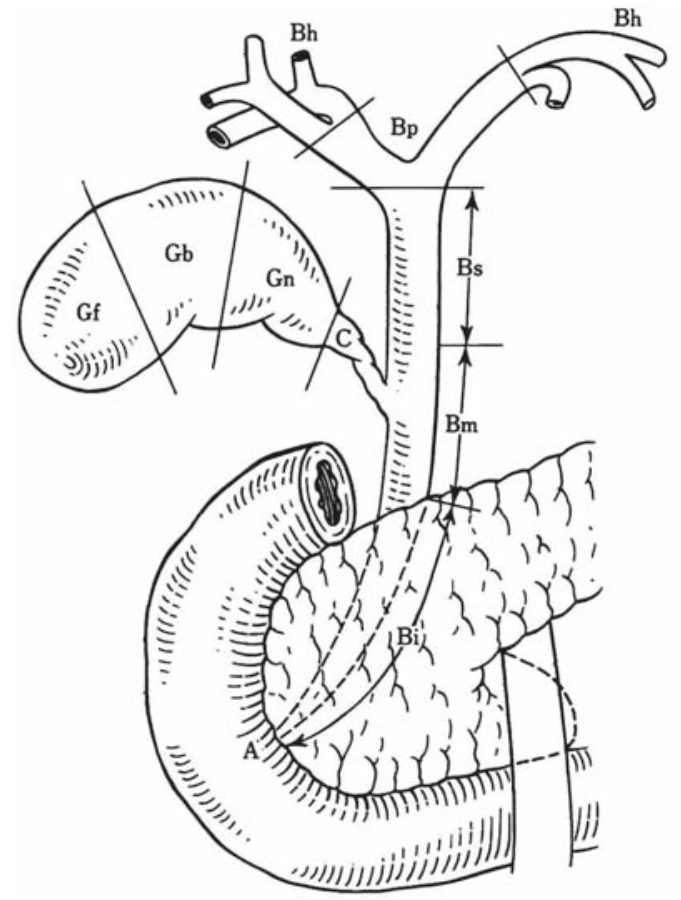

Bh: Intrahepatic bile ducts

Bp : Portal (Hilar) bile duct

Bs : Superior bile duct

Bm : Middle bile duct

$\mathrm{Bi}$ : Inferior bile duct

Gf: Fundus of the gallbladder

$\mathrm{Gb}$ : Body of the gallbladder

$\mathrm{Gn}$ : Neck of the gallbladder

C: Cystic duct

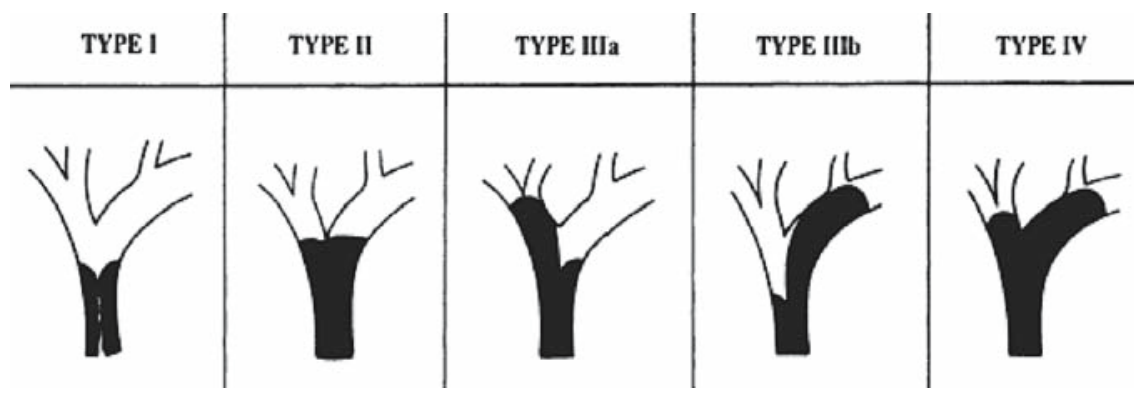

Fig. 2. Anatomical nomenclature of the biliary tract (Japanese Society of Biliary Surgery. Classification of biliary tract carcinoma. Second English edition. Tokyo: Kanehara; 2004), ${ }^{2}$ with permission
Fig. 3. Modified Bismuth-Corlette classification (from Bismuth $\mathrm{H}$, Nakache $\mathrm{R}$, Diamond T. Management strategies in resection for hilar cholangiocarcinoma. Ann Surg 1992;215:31-8.), ${ }^{3}$ with permission 
questions (CQs) regarding surgical treatment for biliary carcinoma. In the responses to the CQs, recommendations for treatment are noted (grades of these recommendations are defined in Table $1^{4}$ ). Also, levels of evidence are given (in parentheses) for findings in reference citations (see definitions of levels in Table $2^{4}$ ).

\section{CQ 1 What unresectable factors are there for biliary} cancer?

\section{Included in these factors are metastases to the liver, lung, peritoneum, and distant lymph node. There is no consensus for local extension.}

As in other malignant diseases, judgment concerning the suitability of surgical resection for biliary cancer should be made on the basis of the evaluation of factors such as general condition, local extension of tumor, and metastasis (lymph node, liver, peritoneum, lung, etc) ${ }^{5,6}$ (level IV). In defining "unresectability," noncurative resection is regarded as "unresectable" even if local resection is technically possible.

Decisions on the tolerance of the patient's general condition to surgical operation differ depending upon the surgical extent of the operative procedure. In biliary tract cancer, major surgery is often needed, including hepatectomy and/or pancreaticoduodenectomy. Careful preoperative evaluation should be made of the functions of the liver, heart, lung, and kidney, including performance status.

Table 1. Strength of recommendations ${ }^{4}$

A, Strongly recommend performing the clinical action

$\mathrm{B}$, Recommend performing the clinical action

$\mathrm{C} 1$, The clinical action may be considered although there is a lack of high-level scientific evidence for its use. May be useful

$\mathrm{C} 2$, Clinical action not definitively recommended because of insufficient scientific evidence. Evidence insufficient to support or deny usefulness

$\mathrm{D}$, Recommend not performing the clinical action
Concerning factors involved in the local extension of hilar cholangiocarcinoma and gallbladder cancer, general evaluation of their extension to the bile duct, portal vein, and hepatic artery should be made. Jarnagin et al. ${ }^{5}$ (level IV) defined unresectable factors involved in the local extension of hilar cholangiocarcinoma as follows:

1. Tumor extension to secondary biliary radicles bilaterally

2. Encasement or occlusion of the main portal vein proximal to its bifurcation

3. Atrophy of one hepatic lobe with contralateral portal vein branch encasement or occlusion

4. Atrophy of one hepatic lobe with contralateral tumor extension to secondary biliary radicles

5. Unilateral tumor extension to secondary biliary radicles with contralateral portal vein branch encasement or occlusion

The definition of unresectable gallbladder cancer is made based on almost the same criteria ${ }^{6}$ (level IV). Concerning hilar cholangiocarcinoma, however, there are reports on more aggressive surgical approaches, such as combined resection and reconstruction of the portal vein and/or hepatic artery ${ }^{7}$ (level IV) and left trisectionectomy ${ }^{8}$ (level IV), but there is no consensus at present on local extension factors in unresectable cases. Also, for middle/lower bile duct cancer, there are reports on combined portal vein resection ${ }^{9}$ (level IV) and combined hepatic artery resection ${ }^{10}$ (level IV), but there is no consensus to date on local extension factors in unresectable cases.

Weber et al. ${ }^{6}$ (level IV) define cases up to N1 with hilar cholangiocarcinoma and gallbladder cancer (metastasis to lymh node in the hepatoduodenal ligament) as resectable cases and N2 cases (metastasis to peripancreatic lymph node) as unresectable cases. However, in Japan, aggressive surgical resection is also being performed for cases of N2 hilar cholangiocarcinoma and gallbladder cancer ${ }^{11,12}$ (level IV). At least as far as gallbladder cancer is concerned, metastasis to the paraaortic lymph node is considered as distant metastasis, similar to liver and peritoneal metastases ${ }^{11,13}$ (level IV). There is a report that, for bile duct cancer, macro-

Table 2. Levels of evidence ${ }^{4}$

Level I

Level II

Level III

Level IV

Level V

Level VI
Systematic review/meta-analysis

One or more randomized clinical trials

Nonrandomized controlled trials

Analytic epidemiology (cohort studies and case-control studies)

Descriptive study (case reports and case-series studies)

Opinions of expert panels and individual experts not based on patient's data 
scopically detectable metastasis to the paraaortic lymph node is also considered as distant metastasis, similar to liver and peritoneal metastases ${ }^{12}$ (level IV).

Most studies report that the metastasis of biliary carcinoma to the liver and lung, peritoneal metastasis, and distant lymph node metastasis (paraaortic and extraperitoneal lymph nodes, etc) are unresectable factors $^{6,11,13,14}$ (level IV). However, there are a few reports showing long-term survival, ${ }^{15}$ which are rare and exceptional cases. Concerning the preoperative diagnosis of peritoneal dissemination and liver metastasis, there is a report suggesting the efficacy of a laparoscopic search ${ }^{6}$ (level IV). However, a preoperative diagnosis of paraaortic lymph node metastasis is difficult to make ${ }^{16}$ (level IV), so at present the most reliable procedure to address this situation is intraoperative biopsy.

\section{CQ 2 Is preoperative portal vein embolization useful for patients with biliary tract cancer requiring hepatectomy?}

\section{Preoperative portal vein embolization may be considered in patients in whom right hepatectomy or more, or hepatectomy with a resection rate exceeding $50-60 \%$ is planned, especially in patients with jaundiced liver. It may contribute to the reduction of complications and surgery-related mortality (recommendation C1).}

Embolization of the right portal vein branch for patients with biliary carcinoma in whom right hepatectomy or more is planned leads to atrophied right liver and a significant increase in future liver remnant volume in 2 or 3 weeks ${ }^{17,18}$ (level IV). On the other hand, it is known that, even in patients without portal vein embolization, the liver remnant becomes larger after right hepatectomy, but it is not clear whether the rate of hypertrophy is similar to that in patients for whom portal vein embolization has been conducted.

According to Nagino et al., ${ }^{19}$ the postoperative inhospital mortality rate was $8.8 \%$ in the world's largest case series study of biliary tract cancer, in which hepatectomy was performed after portal vein embolization (bile duct cancer, 132 cases; gallbladder cancer, 61 cases; total, 193 cases). To be more precise, the postoperative in-hospital mortality rate was $4.5 \%$ in patients with bile duct cancer for whom hepatectomy was conducted after portal vein embolization. This was significantly lower than the mortality rate in patients with gallbladder carcinoma (18\%), and similar to that $(3.7 \%)$ in 136 patients with bile duct cancer in whom the liver resection rate was $50 \%$ or less and no portal vein embolization was carried out, and also the long-term results were similar (level IV).

There are no reports of randomized controlled trials (RCTs) on whether preoperative portal vein embolization contributes to a decreased incidence of postoperative complications such as liver failure and the surgery-related mortality rate, but there is a report of a prospective cohort study by Farges et al. ${ }^{20}$ According to this report, there were no differences in the incidence of postoperative complications according to whether or not preoperative portal vein embolization was performed in 55 patients with hepatocellular carcinoma and metastatic liver cancer for whom right hepatectomy was conducted, but in patients with chronic liver diseases, the incidence of postoperative complications was significantly lower in the group for whom preoperative portal vein embolization was performed (level III).

There are no prospective controlled trials concerning biliary tract cancer, so no sufficient scientific evidence exists. However, in view of the following observations, preoperative portal vein embolization may be considered in patients with jaundiced liver in whom right hepatectomy or more, or hepatectomy with a resection rate exceeding $50 \%-60 \%$ is planned: (1) Based on the decreased incidence of postoperative complications brought about by portal vein embolization in patients with chronic liver disease, in whom hepatic functional reserve was decreased, suggested in the cohort study by Farges et al. ${ }^{20}$ concerning hepatocellular carcinoma and metastatic liver cancer, similar effects may be expected in patients with damaged liver even after the resolution of jaundice. (2) According to a report on 53 patients with hilar cholangiocarcinoma by Hemming et al., ${ }^{21}$ in the group in whom hypertrophy of the future liver remnant was caused by obstruction of the portal vein branch, due to its embolization or cancers, postoperative mortality $(3 \%)$ was lower than that $(21 \%)$ in the group without hypertrophy (level IV). (3) In multiple studies $^{7,22-24}$ reporting the use of portal vein embolization as a routine preoperative procedure for patients undergoing major hepatectomy, no postoperative inhospital death occurred; preoperative portal vein embolization may be considered in patients with a jaundiced liver undergoing hepatectomy with a resection rate of $50 \%-60 \%$ (level IV).

Although there is a report showing that there was no occurrence of complications associated with portal vein embolization itself, ${ }^{19}$ the occurrence of other complications has been reported, such as dislocation of embolization materials outside the planned embolization area, pneumothorax, hemorrhage, portal vein thrombosis, portal hypertension, and transient liver failure. ${ }^{25-27}$ Therefore, indications should be carefully considered, especially in patients with normal liver or those with planned left hepatectomy. 
CQ 3 Are there effective measures of hepatic functional reserve in the jaundiced liver?

Measurement of the future liver remnant volume by computed tomography (CT) is useful (recommendation B). The indocyanine green (ICG) clearance test is useful in assessing hepatic functional reserve after relief of jaundice not associated with cholangitis (recommendation C1).

In patients with biliary tract cancer, the association of jaundiced liver is often observed. As described in CQ2, in patients undergoing major hepatectomy such as right hepatectomy, or more, portal vein embolization may need to be considered in order to increase the future liver remnant volume. CT volumetry before and after portal embolization is useful to assess its effect.

There are reports on the ICG clearance test and the galactose tolerance test after relief of jaundice as a method of preoperative assessment of hepatic functional reserve. However, in terms of the occurrence of postoperative complications, no significant difference has been observed in the efficacy of these tests. Concerning the development of postoperative liver failure, the result of the ICG clearance test is considered to serve as a predictive factor.

For predicting postoperative death and the development of liver failure in patients with liver cirrhosis, the usefulness of preoperative assessment of hepatic functional reserve according to the ICG retention rate at $15 \mathrm{~min}$ is widely recognized ${ }^{28}$ (level IV). Also, more appropriate decision-making is made possible by measuring the ratio of the future remnant liver volume to the total liver volume ${ }^{29}$ (level IV). Furthermore, Nagino et al $^{30}$ reported that four factors: glucose tolerance, the presence or absence of cholangitis and pancreaticoduodenectomy, and the ICG clearance rate were involved (level IV). The ICG retention rate at $15 \mathrm{~min}$ is generally used in the preoperative assessment of hepatic functional reserve, but the number of patients with jaundiced livers in the reports on the preoperative assessment of hepatic functional reserve is smaller than the numbers of those with normal and cirrhotic livers. There is also a report that the occurrence of cholangitis prior to resection had unfavorable impacts on postoperative results even in patients with good hepatic functional reserve ${ }^{31}$ (level IV).

It is considered that the best clinical method at present includes the measurement of the future liver remnant volume with the use of $\mathrm{CT}$ in the first place, and the overall assessment of hepatic functional reserve based on the ICG retention rate at $15 \mathrm{~min}$ after the relief of jaundice, and determination of the presence or absence of cholangitis. There is a report that reviewed the ICG clearance test on the basis of bile excretion volume ${ }^{32}$ (level IV). According to this report, this test may be helpful in lobar functional assessment, but it is not always suitable because drainage is often carried out for one lobe. The galactose tolerance test is also helpful in assessing hepatic functional reserve in the jaundiced liver, but its evidence level is low $^{33}$ (level IV). There is a possibility that, as cases in this field accumulate, a test enabling lobar functional assessment ${ }^{34}$ (level IV), such as ${ }^{99 \mathrm{~m}} \mathrm{Tc}$-galactosyl human serum albumin (GSA) scintigraphy, may become effective.

CQ 4 Is extrahepatic bile duct resection alone recommended for upper/middle bile duct cancer?

Although hepatectomy and/or pancreaticoduodenectomy are preferable for curative resection, extrahepatic bile duct resection alone is considered in patients for whom it is judged that curative resection can be achieved after a strict diagnosis of the local extension of the cancer (recommendation C2).

Standard operative procedures in the surgical treatment of bile duct cancer are bile duct resection plus hepatectomy for hilar/upper bile duct carcinoma and pancreaticoduodenectomy (PD) for middle/lower bile duct cancer. Many bile duct cancers are associated with lymph node metastasis and perineural invasion, in addition to longitudinal and extramural extensions, and tumor remnant in the resection stump and lymph node metastasis are reported to be important prognostic factors after the resection of bile duct cancer ${ }^{35}$ (level IV). In the common type of bile duct cancer, a negative resection stump is difficult to secure by means of bile duct resection plus local lymph node dissection alone, and lymph node dissection itself is also assumed to be often insufficient. However, if the cancer is a localized type without lymph node metastasis and perineural invasion, curative resection may be achieved, theoretically, with the use of extrahepatic bile duct resection (BDR) alone. ${ }^{36}$

Jang et al. ${ }^{37}$ performed BDR for 27 patients with upper/middle bile duct cancer and found a 5-year survival rate of $28.0 \%$ for these patients $(7 / 25)$. They reported that no significant difference was observed between PD/hepatectomy and BDR alone, the survival rate of $\mathrm{PD}$ in middle/lower bile duct cancer being $30.1 \%(31 / 103)$ and that of BDR with hepatectomy in upper/middle bile duct cancer being 47.8\%(11/23). According to their report, prognostic factors after BDR are the type of cancer and lymph node metastasis. Bile duct resection is recommended for T1 lesions with histological findings of the papillary type or well- 
differentiated adenocarcinoma, especially in patients with poor general condition (level IV).

Good indications for BDR include a papillary type of lesion limited to the upper/middle bile duct without apparent lymph node metastasis, in which the resection stump should be confirmed to be negative by intraoperative pathological diagnosis. ${ }^{38}$ For a nodular/ invasive type of tumor, hepatectomy or PD is preferred as a rule, ${ }^{7,38}$ and BDR may be recommended in patients who are judged to be intolerant of hepatectomy or PD.

CQ 5 Is combined caudate lobe resection required for hilar/upper bile duct carcinoma?

Combined caudate lobe resection is recommended in most cases of hilar/upper bile duct carcinoma (recommendation C1).

There are no RCTs reporting on the efficacy of caudate lobe resection for hilar cholangiocarcinoma. Mizumoto et al. ${ }^{39}$ (level IV) reported that, in 11 of 24 patients with resected hilar cholangiocarcinoma, tumor invasion was found in the caudate branches or parenchyma, thus demonstrating the importance of caudate resection. Also, Nimura et al. ${ }^{40}$ (level IV) performed combined caudate resection in 45 of 55 patients with resected hilar cholangiocarcinoma and achieved a 5-year survival rate of $40.5 \%$ for 43 patients who underwent curative resection, concluding that caudate resection is the hepatectomy type of choice.

The present situation in Japan is that combined caudate resection is being widely performed for hilar cholangiocarcinoma ${ }^{7,8,22-24}$ (level IV). This resection is being performed after consideration of these reports and consideration of the anatomical characteristics of caudate branches that directly join the hepatic hilar bile duct. ${ }^{41,42}$ There is a report that, in the absence of cancer invasion to the caudate bile duct branch in patients with the Bismuth I type of carcinoma, no difference was found in prognosis between patients who underwent hepatectomy including caudate resection and those in whom curative resection was achieved by hepatic hilar resection preserving the caudate lobe ${ }^{36}$ (level IV).

Concerning reports from other countries, we have found an extremely small number of reports that discuss the benefits or harms of combined caudate resection for hilar cholangiocarcinoma ${ }^{43}$ and, in contrast to the efforts in Japan, there is only a limited amount of literature discussing caudate resection in review articles. ${ }^{44-46}$ However, at the present time, Italian and Korean groups have reported improved survival rates with the use of combined caudate resection, and they have stressed the necessity of caudate resection, considering the lack of established diagnostic methods to judge invasion to the caudate bile duct branch ${ }^{47,48}$ (level IV).

CQ 6 Should combined portal vein resection be performed for patients with portal vein invasion?

Combined portal vein resection may be performed, because the prognosis of patients treated with combined portal vein resection is significantly better than that of unresected patients (recommendation C1).

Ebata et al. ${ }^{49}$ reported combined portal vein resection in 52 of 160 patients with resected hilar cholangiocarcinoma. In patients with combined portal vein resection, the cancer was more advanced, but no difference was observed in mortality rates between patients with and without portal vein resection. The 5-year survival rates were $9.9 \%$ and $36.8 \%$, respectively, being significantly lower in patients with portal vein resection. There was no difference in prognosis on the basis of the presence or absence of pathological portal vein invasion. By multivariate analysis, it was found that factors involved in prognosis were the degree of differentiation and the presence or absence of lymph node metastasis and macroscopic portal vein invasion. According to the authors, portal vein resection contributed to long-term survival in a small number of patients with advanced cancer (level IV).

Miyazaki et al. $^{50}$ conducted combined portal vein resection in 41 of 161 patients who underwent resection of hilar cholangiocarcinoma. The 5-year survival rates in patients with curative resection were $25 \%$ and $41 \%$ for those receiving combined portal vein resection and those receiving nonvascular resection, respectively. Although the prognosis was significantly poorer in patients with combined vascular resection, it was better compared with that in unresectable patients. So, these authors reported that combined portal vein resection was of significance (level IV). The same group (Suzuki et $\mathrm{l}^{51}$ ) also reported on the usefulness and safety of left renal vein graft in the reconstruction of the portal vein and inferior vena cava (level IV).

Kondo et al. ${ }^{52}$ reported no postoperative in-hospital deaths in patients receiving combined portal vein resection, and they noted that it could be carried out safely; no difference was found in postoperative morbidity and mortality between these patients and patients with noncombined resection ${ }^{7}$ (level IV).

Hemming et al $^{53}$ conducted combined portal vein resection in 26 of 60 patients who underwent curative resection for hilar cholangiocarcinoma. They found no 
significant difference between patients with and without portal vein resection in the occurrence of complications and mortality rates, frequency of positive resection stump, and 5-year survival rates (patients with portal vein resection, $39 \%$; patients without portal vein resection, $41 \%)$. Multivariate analysis showed that the only factor responsible for prognosis was the presence of a positive resection stump. According to their report, portal vein resection is a safe procedure and may contribute to longer survival (level IV).

Portal vein invasion in hilar cholangiocarcinoma is one of the factors that determines resectability and the method of resection. ${ }^{5}$ Several studies have reported cases which became resectable with the use of combined portal vein resection. It is suggested that the prognosis of resected patients was better than that of unresectable cases. However, many of the patients compared were unresectable not necessarily because of portal vein invasion. Therefore, the strength of recommendation for resection was graded as category $\mathrm{C} 1$ due to the lack of sufficient scientific evidence to clarify the significance of combined portal vein resection ${ }^{49,50,53,54}$ (level IV).

CQ 7 What are the prognostic factors after resection of bile duct cancer?

Included in these factors are positive surgical margins, especially in the ductal stump; lymph node metastasis; perineural invasion; and combined vascular resection due to portal vein and/or hepatic artery invasion.

Kondo et al. ${ }^{7}$ summarized reports of multivariate analysis of prognostic factors after resection of hilar cholangiocarcinoma after resection. According to their study, 9 of 11 reports suggested the presence or absence of R0 (curative resection), as the factor with the greatest impact on prognosis. Because the prognosis in curatively resected patients is significantly better than that in patients with noncurative resection and unresectable patients, achieving curative resection is of great importance. Particularly, a large number of studies ${ }^{43,55-59}$ have suggested the absence of cancer remnant in the resection stump and surgical margin as an important factor that contributes to achieving a good prognosis (level IV). However, a cancer remnant in the mucosa of the ductal stump has been said by others to have no impact on prognosis ${ }^{60}$ (level IV).

In patients who have undergone curative resection, the presence or absence of lymph node metastasis has a large impact on their prognosis ${ }^{2,56-58}$ (level IV). There is a report ${ }^{56}$ (level IV) suggesting that no correlation exists between the degree of lymph node metastasis and prognosis, and that the important factor in determining the prognosis is the presence or absence of lymph node metastasis. Furthermore, there is a report indicating that the prognosis of patients with lymph node metastasis is generally poor, but for those in whom metastasis is limited to the regional lymph node, the prognosis is not always poor if systematic lymph node dissection is performed $^{59}$ (level IV). Also, on the basis of results of univariate and multivariate analyses, the presence or absence of lymph node metastasis is thought to be an important prognostic factor after resection.

There is a report suggesting that perineural invasion is also an important mode of extension in bile duct cancer and that its presence or absence is a factor involved in the prognosis after resection of this cancer ${ }^{61}$ (level IV).

In multivariate analysis that included patients who underwent combined vascular resection due to the invasion of the portal vein and/or hepatic artery, vascular resection was reported as one of the negative prognostic factors $^{49,62}$ (level IV).

The curative resectability of middle/lower bile duct cancer as well as hilar cholangiocarcinoma has the largest impact on prognosis. It is reported that the absence of a cancer remnant in the resection stump and surgical margin is of utmost importance, but that lymph node metastasis is an important prognostic factor as well $^{35,63}$ (level IV).

There are reports that found a correlation between perineural invasion and prognosis ${ }^{43,61}$ (level IV), while another report failed to find such a correlation ${ }^{35}$ (level IV).

Thus, in bile duct cancer, an important factor that has a large impact on prognosis is whether the resection was curative or not; therefore, a detailed examination of the resected sample is of significance. Patients with positive surgical margins or with positive lymph nodes have a higher possibility of recurrence. Strict followup and postoperative adjuvant therapy should be considered.

CQ 8 Should open cholecystectomy be performed instead of laparoscopic cholecystectomy for patients with suspected gallbladder cancer?

For patients with suspected gallbladder cancer, laparoscopic cholecystectomy is not recommended, and open cholecystectomy should be performed as a rule (recommendation $\mathrm{C1}$ ).

Laparoscopic cholecystectomy (LC) is the first choice for cholecystolithiasis, and there is a tendency to extend its indication to patients with suspected gallbladder 
cancer. However, in LC, the dissection plane on the liver side is within the subserosal layer. If the lesion has extended beyond the muscular layer, a cancer remnant may result. If a mucosal carcinoma in the RokitanskyAshoff sinus (RAS) extending beyond the muscular layer exists on the liver side, a cancer remnant may also result. Even in whole-layer cholecystectomy, in which the hepatic parenchyma is exposed, the subserosal layer may be exposed if a subserosal cancer has extended deep into the layer. The resectable extent with LC is rather limited and, at least, LC should not be used for a lesion on the liver side.

The methods and procedures for lymph node dissection and combined resection of the liver for gallbladder cancer have not been established as laparoscopic procedures. Therefore, these steps are not recommended at present.

In a study of 123 patients with polypoid lesions, carried out by Yeh et al. ${ }^{64}$ only $2(2.1 \%)$ of the 28 neoplastic lesions were less than $10 \mathrm{~mm}$ in diameter and all of the 7 malignant lesions were $15 \mathrm{~mm}$ or more. On the basis of mutlivariate analysis including these data, it was shown that polyps $10 \mathrm{~mm}$ or more in diameter are neoplastic, and it is probable that polyps of $15 \mathrm{~mm}$ or more are malignant (level IV). Therefore, polypoid lesions with a largest diameter of less than $10 \mathrm{~mm}$ may be considered in indications for LC, but for lesions larger than $10 \mathrm{~mm}$, careful attention should be paid to the suspected gallbladder cancer, especially in the case of lesions on the liver side.

When LC is applied for gallbladder cancer, port-site recurrence (PSR) and peritoneal recurrence occurring as a result of bile leak associated with gallbladder injury are serious problems. Wakai et al. ${ }^{65}$ reported, in their study of LC, that, of 28 patients with gallbladder cancer, gallbladder injury occurred in $25 \%$, of whom PSR or local recurrence developed in $43 \%$, and the survival rate was significantly lower in patients with gallbladder injury (level IV). Ouchi et al. ${ }^{66}$ found that gallbladder perforation occurred in $20 \%$ of patients with gallbladder cancer, and that the prognosis was significantly poorer in patients with perforation than in patients without perforation. In their report, the recurrence rate in patients with and without gallbladder perforation was $27 \%$ and $14 \%$, respectively, being significantly higher in the former group. There are also reports concerning cases of intraepithelial carcinoma ${ }^{67}$ and mucosal cancer ${ }^{68}$ in which early death occurred due to peritoneal dissemination as a result of gallbladder perforation (level V).

It is reported that the incidence of PSR after LC was $11 \%-16 \%,{ }^{65,69-71}$ and the time to onset was $6-10$ months. ${ }^{69-71}$ There is a report showing that the prognosis of patients with PSR was poor; all the patients had associated peritoneal dissemination, and the median survival period was 19 months $^{71}$ (level IV). There are studies comparing the incidence of PSR with that of wound recurrence in patients who underwent open cholecystectomy (OC). According to these studies, the incidence (LC: OC) was $15 \%: 6.5 \%,{ }^{72}$ and $11 \%: 4 \%,{ }^{73}$ respectively (level IV). It is thought that, in addition to intraoperative bile leak, such factors as the biological properties of the tumor, tumor stage, operative maneuvers, and the impact of pneumoperitoneal pressure and carbon dioxide on tumor cells and the living body are involved in the onset of PSR. ${ }^{69}$

LC can now be safely performed for cholecystolithiasis because of technical improvements and advances in instrumentation. It is speculated that, theoretically, LC can be indicated for early gallbladder cancer with intramural invasion up to the muscular layer, but the accurate preoperative diagnosis of the depth of mural invasion is difficult even with full use of ultrasonography, CT, and endoscopic ultrasonography (EUS) ${ }^{74}$ (level IV). Taking into account intraoperative bile leak and the incidence of PSR, LC is not recommended for suspected gallbladder cancer.

\section{CQ 9 Is an additional resection required when gallbladder cancer invading the subserosal layer or deeper has been detected after simple cholecystectomy?}

\section{An additional resection should be considered (recommendation C1).}

With the recent progress in diagnostic imaging, a diagnosis of advanced gallbladder cancer can be made prior to operation in many patients. But it is reported that the frequency of diagnosis of gallbladder cancer being made serendipitously by postoperative pathological examinations of gallbladders resected for gallstones is approximately $1 \% .{ }^{67,75,76}$ In patients in whom a diagnosis of $\mathrm{m}$ or mp cancer (see Table 3 for definitions of depth of mural invasion of gallbladder cancer) has been made by pathological examination of the entire gallbladder, an additional resection is not necessary as a rule if the

Table 3. Depth of mural invasion of gallbladder cancer ${ }^{a}$

\begin{tabular}{ll}
\hline $\mathrm{m}$ & Invasion limited to the mucosa \\
$\mathrm{mp}$ & Invasion limited to the muscularis propria \\
$\mathrm{Ss}$ & Invasion limited to the subserosa \\
$\mathrm{se}$ & Invasion of the serosal surface \\
$\mathrm{si}$ & $\begin{array}{l}\text { Invasion beyond the serosa and invasion of other } \\
\text { organs or structures }\end{array}$ \\
\hline
\end{tabular}

${ }^{\mathrm{a}}$ Japanese Society of Biliary Surgery (JSBS). Classification of biliary tract carcinoma, Second English edition 2004. ${ }^{2}$ 
cystic duct stump is negative $\mathrm{e}^{77,78}$ (level IV). However, in gallbladder cancer invading the subserosal layer, high rates of vascular and perineural invasion ${ }^{79-81}$ and positive lymph node metastasis $(40 \%-50 \%)$ are observed. ${ }^{81-85}$

Although there is no RCT concerning additional resection, there are retrospective studies reporting that, in patients with gallbladder cancer invading the subserosal layer or deeper, prognosis is significantly better in the group for whom additional resection has been performed compared with the group who received simple cholecystectomy alone $e^{66,77,86,87}$ (level IV). Thus, it is thought that in gallbladder cancer invading the subserosal layer or more, a curative second operation including hepatectomy and lymphadenectomy, when necessary, should be considered ${ }^{88-91}$ (level V). However, in patients with advanced extracystic extension, the successful resection rate in second operations and the 5-year survival rate after resection are low and prognosis is poor $^{77,85,92}$ (level IV).

\section{CQ 10 What are the prognostic factors after resec-} tion of gallbladder cancer?

Included in these factors are depth of mural invasion; lymph node metastasis; extramural extension,, especially into the hepatoduodenal ligament; perineural invasion; and the degree of curability.

The 5-year survival rate of patients after the resection of advanced gallbladder cancer is $33 \%-65 \%$ for International Union Against Cancer (UICC) stage III and $8 \%-25 \%$ for stage IV, and its prognosis is clearly poorer compared with that of stage I (79\%-91\%) and stage II $(64 \%-85 \%)^{82,92-94}$ (level IV). See Table 4 for UICC staging of gallbladder cancer.

There are many studies concerning prognostic factors after the resection of gallbladder cancer, and significant

Table 4. UICC staging of gallbladder cancer

\begin{tabular}{llll}
$\begin{array}{c}\text { Stage grouping } \\
\text { Stage 0 }\end{array}$ & Tis & N0 & M0 \\
Stage IA & T1 & N0 & M0 \\
Stage IB & T2 & N0 & M0 \\
Stage IIA & T3 & N0 & M0 \\
Stage IIB & T1, 2, 3 & N1 & M0 \\
Stage III & T4 & Any N & M0 \\
Stage IV & Any T & Any N & M1 \\
\hline
\end{tabular}

TNM Classification summary

T1, gallbladder wall; T1a, lamina propria; T1b, muscle; T2, perimuscular connective tissue; $\mathrm{T} 3$, serosa, one organ and/or liver; T4, portal vein, hepatic artery, or two or more extrahepatic organs; N1, regional lymph node metastasis prognostic factors include UICC stage, lymph node metastasis, depth of invasion, bile duct invasion, hepatic invasion, hepatoduodenal ligament invasion, perineural invasion, histological type, and curability ${ }^{74,95,96}$ (level IV).

The UICC stage shows the degree of cancer extension and it is a matter of fact that more advanced cancer has a poorer prognosis. Of the $\mathrm{TN}$ factors, the most important prognostic factor in advanced gallbladder cancer is lymph node metastasis, which is also reported to be an independent prognostic factor on the basis of multivariate analysis ${ }^{92,95,97}$ (level IV). In reports from Western countries, most of the long-term survivors with T3, T4 gallbladder cancer had N0 disease. ${ }^{92}$ On the other hand, there is a report showing that, in Japan, the prognosis of $\mathrm{N} 1$ patients (metastasis limited to the lymph node within the hepatoduodenal ligament) was improved by lymph node dissection and that no significant difference was found between the 5-year survivals of N0 patients $(66 \%)$ and N1 patients $(53 \%)^{98}$ (level IV). There is a report which shows that, for $\mathrm{N} 2$ patients (metastasis to the lymph node around the pancreas head), the 5 -year survival rate remains at $16 \%$, which is poorer compared with that of $\mathrm{N} 1{ }^{98}$ Also, there is a study which reports that no difference was observed in the 5year survival rates between N1 and N2 disease and that N3 (paraaortic lymph node metastasis) disease had a poor prognosis ${ }^{11}$ (level IV).

Another important prognostic factor, as well as lymph node metastasis, is thought to be hepatoduodenal ligament invasion. In Japan, it has been reported that hepatoduodenal ligament invasion is an important prognostic factor $^{99}$ (level IV), while reports mentioning hepatoduodenal ligament invasion are rare in Western countries. The local extension of gallbladder cancer is a $\mathrm{T}$ factor, such as findings of depth of mural invasion, bile duct invasion, and liver invasion, but hepatoduodenal ligament invasion is a finding that shows not only bile duct invasion but also cancer cell infiltration into the interstitial tissues within the hepatoduodenal ligament. As a histological finding of gallbladder cancer, perineural invasion has also been reported to be a factor responsible for poor prognosis, ${ }^{98}$ and this invasion is closely associated with bile duct invasion. It has also been made clear that perineural invasion is an independent factor responsible for poor prognosis ${ }^{95,97}$ (level IV).

On the basis of univariate analysis concerning UICC stage I-IV gallbladder cancer, it was reported that the degree of histological differentiation of gallbladder cancer was also a significant prognostic factor ${ }^{74,95}$ (level IV). However, another study of this disease notes that, except for that in stage I, the degree of differentiation is not always an important prognostic factor. ${ }^{92}$ Most T1 cancers are papillary adenocarcinomas or well differentiated tubular adenocarcinomas without lymph node 
metastasis, and the prognosis is good, whereas in T3-4 cancers, the prognosis is poor. The difference in prognosis between T1 and T3-4 cancers is possibly caused by the finding that even well-differentiated tubular adenocarcinomas in the latter are frequently associated with lymph node metastasis. ${ }^{98}$

Also, similar to findings in other malignant cancers, the degree of curability after surgical resection is a prognostic factor ${ }^{92}$ (level IV). Gallbladder cancer shows a variety of modes of spread to the surrounding organs, such as lymph node metastasis, liver invasion, hepatoduodenal ligament invasion, duodenal invasion, and transverse colon invasion, so the selection of surgical procedures according to the modes of tumor spread and the achievement of R0 are considered to be of great significance $^{14,15,99}$ (level IV). Furthermore, there are a few reports demonstrating that, even for advanced gallbladder cancer presumed to have poor prognosis due to lymph node metastasis (N2) or hepatoduodenal ligament invasion, long-term survival may be achieved by $\mathrm{R} 0$ resection with the use of hepatic resection plus PD (extended lymph node dissection) ${ }^{100,101}$ (level IV). Longterm survival is not expected in patients who have had $\mathrm{R} 1$ or $\mathrm{R} 2$ resections.

CQ 11 For which type of ampullary carcinoma is limited operation recommended?

\section{Pancreaticoduodenectomy (PD) is indicated for ampullary carcinoma in general, but limited operation is also indicated in patients with carcinoma in adenoma (recommendation C1).}

Pancreaticoduodenectomy (PD) remains as a standard surgical procedure for ampullary carcinoma, and improved operative techniques and perioperative management have contributed to better results. It is reported, on the basis of pathological examination, that the rate of lymph node metastasis is low in ampullary cancers not extending beyond the sphincter of Oddi ${ }^{102,103}$ (level IV). Under these circumstances, there have been many reports in recent years on a variety of limited operation as curative surgery for ampullary cancer. However, there are no reports of RCTs concerning limited operation and standard operation. Furthermore, resections with curative intent and palliative resections selected for patients in poor general conditions are intermingled in these reports, thus making an accurate assessment of treatment results difficult. Knox and Kingston ${ }^{104}$ reported, in their case-series study (level IV), that the survival rate after local resection was slightly better than that after PD. Goldberg et al. ${ }^{105}$ and Sharp et al. ${ }^{106}$ (level IV) reported that the results of local resection were safe and as good as those of PD. On the other hand, there is a report demonstrating that a higher rate of recurrence was observed for limited surgery than was observed for PD. Also, Branum et al. ${ }^{107}$ (level IV) reported that cancer recurred in six of eight patients with local resection.

Concerning indications for limited surgery, Klein et al. ${ }^{108}$ (level IV) reported that local resection was indicated for adenoma or T1N0 tumors, whereas Paramythiotis et al. ${ }^{109}$ (level IV) suggested that it was indicated for adenoma with high-grade dysplasia and villous, tubulovillous, or tubular adenomas less than $2 \mathrm{~cm}$ in size. Beger et al. ${ }^{110}$ (level IV) reported that carcinoma was found in $26 \%$ of resected patients with a preoperative diagnosis of papillary adenoma, thus concluding that patients with pTis, pT1, N0, M0, G1, or G2 disease were indicated for limited surgery and that local lymph node dissection should be performed for these patients.

At present, making a diagnosis of cancer invasion to the sphincter of Oddi is difficult, so no consensus has been reached on the usefulness of local resection in patients with a definite diagnosis of malignancy. To apply limited surgery for this disease, improved diagnostic precision and the results of RCTs will be necessary, but at present, indications for limited surgery are adenoma or cancer in adenoma with a low possibility of invasion of the sphincter of Oddi. Also, endoscopic ampullectomy is being attempted for adenoma.

CQ 12 What prognostic factors are there after resection of ampullary carcinoma?

Included in these factors are lymph node metastasis, pancreas invasion, and perineural invasion.

Among biliary tract cancers, the prognosis of ampullary carcinoma is relatively good, but its prognosis is slightly poorer than that of digestive tract cancer. In patients without distant metastasis, curative resection is of the utmost importance, and such resection can be carried out for most ampullary carcinomas.

In biliary tract cancers, lymph node metastasis and perineural invasion are important modes of extension. ${ }^{111}$ There are many reports demonstrating that the presence or absence of lymph node metastasis has a large impact on prognosis in ampullary carcinoma ${ }^{2,110,112-114}$ (level IV). There is a report indicating that the frequency of perineural invasion is lower than that in bile duct cancer, but that this invasion is also an important prognostic factor ${ }^{115}$ (level IV).

Pancreatic invasion is also a significant mode of extension, and the prognosis of patients with pancreatic invasion is poorer than that of patients with no pancreatic 
invasion $^{115}$ (level IV). Furthermore, there is a report indicating that the gross tumor appearance is also an important prognostic factor ${ }^{113}$ (level IV), but this factor is closely correlated with lymph node metastasis.

In summary, detailed investigation of the extension of ampullary carcinoma makes possible the prediction of a high risk of recurrence, and strict follow-up and adjuvant therapy after resection should be considered in such high-risk patients.

Acknowledgment. We would like to express our deep gratitude to the members of the the Japanese Association of Biliary Surgery, the Japanese Society of HepatoBiliary-Pancreatic Surgery, and the Japan Society of Clinical Oncology, who provided us with great support and guidance in the preparation of the Guidelines. This process was conducted as part of the Integrated Research Project for Assessing Medical Technology 2005 and 2006 sponsored by the Japanese Ministry of Health, Labour, and Welfare.

We truly appreciate the following active working members who developed the draft of the evidencebased clinical practice Guidelines for the treatment of biliary tract cancer (Japanese version, 2007): Masahiro Kai (Miyazaki), Yasutoshi Kimura (Sapporo), Shigeaki Sawada (Toyama), Hiroaki Shimizu (Chiba), Hisatoshi Nakagawara (Kanazawa), Kohei Nakachi (Kashiwa), and Hiroyuki Yoshitome (Chiba). We also appreciate very much the following members who reviewed and approved the final Japanese version of the guidelines: Hiromitsu Saisyo (Ichikawa), Munemasa Ryu (Chiba), Satoru Shikata (Kyoto), and Yuji Nimura (Nagoya).

\section{References}

1. Nagakawa T, Kayahara M, Ikeda S, Futakawa, S, Kakita A, Kawarada H, et al. Biliary tract cancer treatment: results from the Biliary Tract Cancer Statistics Registry in Japan. J Hepatobiliary Pancreat Surg 2002;9:569-75.

2. Japanese Society of Biliary Surgery. Classification of biliary tract carcinoma. Second English edition. Tokyo: Kanehara; 2004.

3. Bismuth H, Nakache R, Diamond T. Management strategies in resection for hilar cholangiocarcinoma. Ann Surg 1992;215: $31-8$.

4. Takada T, Miyazaki M, Miyakawa S, Tsukada K, Nagino M, Kondo S, et al. Purpose, use, and preparation of clinical practice guidelines for the management of biliary tract and ampullary carcinomas. J Hepatobiliary Pancreat Surg 2008;15:2-6.

5. Jarnagin WR, Fong Y, DeMatteo RP, Gonen M, Burke EC, Bodniewicz BS J, et al. Staging, resectability, and outcome in 225 patients with hilar cholangiocarcinoma. Ann Surg 2001;234: 507-17.

6. Weber SM, DeMatteo RP, Fong Y, Blumgart LH, Jarnagin WR. Staging laparoscopy in patients with extrahepatic biliary carcinoma: analysis of 100 patients. Ann Surg 2002;235:392-9.

7. Kondo S, Hirano S, Ambo Y, Tanaka E, Okushiba S, Morikawa $\mathrm{T}$, et al. Forty consecutive resections of hilar cholangiocarci- noma with no postoperative mortality and no positive ductal margins: results of a prospective study. Ann Surg 2004;240: 95-101.

8. Nagino M, Kamiya J, Arai T, Nishio H, Ebata T, Nimura Y. "Anatomic" right hepatic trisectionectomy (extended right hepatectomy) with caudate lobectomy for hilar cholangiocarcinoma. Ann Surg 2006;243:28-32.

9. Roder JD, Stein HJ, Siewert JR. Carcinoma of the periampullary region: who benefits from portal vein resection? Am J Surg 1996;171:170-4.

10. Kondo S, Hirano S, Ambo Y, Tanaka E, Kubota T, Katoh H. Arterioportal shunting as an alternative to microvascular reconstruction after hepatic artery resection. Br J Surg 2004;91:24851.

11. Kondo S, Nimura Y, Hayakawa N, Kamiya J, Nagino M, Uesaka K. Regional and para-aortic lymphadenectomy in radical surgery for advanced gallbladder carcinoma. Br J Surg 2000; 87:418-22.

12. Kitagawa Y, Nagino M, Kamiya J, Uesaka K, Sano T, Yamamoto $\mathrm{H}$, et al. Lymph node metastasis from hilar cholangiocarcinoma: audit of 110 patients who underwent regional and paraaortic node dissection. Ann Surg 2001;233:385-92.

13. Shimada H, Endo I, Togo S, Nakano A, Izumi T, Nakagawara G. The role of lymph node dissection in the treatment of gallbladder carcinoma. Cancer 1997;79:892-9.

14. Chijiiwa K, Kai M, Nagano M, Hiyoshi M, Ohuchida J, Kondo K. Outcome of radical surgery for stage IV gallbladder carcinoma. J Hepatobiliary Pancreat Surg 2007;14:345-50.

15. Nishio H, Nagino M, Ebata T, Yokoyama Y, Igami T, Nimura Y. Aggressive surgery for stage IV gallbladder carcinoma; what are the contraindications? J Hepatobiliary Pancreat Surg 2007; 14:351-7.

16. Noji T, Kondo S, Hirano S, Tanaka E, Ambo Y, Kawarada Y, et al. CT evaluation of paraaortic lymph node metastasis in patients with biliary cancer. J Gastroenterol 2005;40:739-43.

17. Makuuchi M, Thai BL, Takayasu K, Takayama T, Kosuge T, Gunvén P, et al. Preoperative portal embolization to increase safety of major hepatectomy for hilar bile duct carcinoma: a preliminary report. Surgery 1990;107:521-7.

18. Nagino M, Nimura Y, Kamiya J, Kondo S, Uesaka K, Kin Y, et al. Changes in hepatic lobe volume in biliary tract cancer patients after right portal vein embolization. Hepatology 1995; 21:434-9.

19. Nagino M, Kamiya J, Nishio H, Ebata T, Arai T, Nimura Y. Two hundred forty consecutive portal vein embolizations before extended hepatectomy for biliary cancer: surgical outcome and long-term follow-up. Ann Surg 2006;243:364-72.

20. Farges O, Belghiti J, Kianmanesh R, Regimbeau JM, Santoro R, Vilgrain V, et al. Portal vein embolization before right hepatectomy: prospective clinical trial. Ann Surg 2003;237:208-17.

21. Hemming AW, Reed AI, Fujita S, Foley DP, Howard RJ. Surgical management of hilar cholangiocarcinoma. Ann Surg 2005; 241:693-9.

22. Seyama Y, Kubota K, Sano K, Noie T, Takayama T, Kosuge T, et al. Long-term outcome of extended hemihepatectomy for hilar bile duct cancer with no mortality and high survival rate. Ann Surg 2003;238:73-83.

23. Kawasaki S, Imamura H, Kobayashi A, Noike T, Miwa S, Miyagawa S. Results of surgical resection for patients with hilar bile duct cancer: application of extended hepatectomy after biliary drainage and hemihepatic portal vein embolization. Ann Surg 2003;238:84-92.

24. Sano T, Shimada K, Sakamoto Y, Yamamoto J, Yamasaki S, Kosuge T. One hundred two consecutive hepatobiliary resections for perihilar cholangiocarcinoma with zero mortality. Ann Surg 2006;244:240-7.

25. Kodama Y, Shimizu T, Endo H, Miyamoto N, Miyasaka K. Complications of percutaneous transhepatic portal vein embolization. J Vasc Interv Radiol 2002;13:1233-7. 
26. Di Stefano DR, de Baere T, Denys A, Hakime A, Gorin G, Gillet M. Preoperative percutaneous portal vein embolization: evaluation of adverse events in 188 patients. Radiology 2005;234: 625-30.

27. Madoff DC, Abdalla EK, Gupta S, Wu TT, Morris JS, Denys A, et al. Transhepatic ipsilateral right portal vein embolization extended to segment IV: improving hypertrophy and resection outcomes with spherical particles and coils. J Vasc Interv Radiol 2005;16:215-25.

28. Miyagawa S, Makuuchi M, Kawasaki S, Kakazu T. Criteria for safe hepatic resection. Am J Surg 1995;169:589-94.

29. Kubota K, Makuuchi M, Kusaka K, Kobayashi T, Miki K, Hasegawa K, et al. Measurement of liver volume and hepatic functional reserve as a guide to decision-making in resectional surgery for hepatic tumors. Hepatology 1997;26:1176-81.

30. Nagino M, Nimura Y, Hayakawa N, Kamiya J, Kondo S, Sasaki $\mathrm{R}$, et al. Logistic regression and discriminant analyses of hepatic failure after liver resection for carcinoma of the biliary tract. World J Surg 1993;17:250-5.

31. Kanai M, Nimura Y, Kamiya J, Kondo S, Nagino M, Miyachi M, et al. Preoperative intrahepatic segmental cholangitis in patients with advanced carcinoma involving the hepatic hilus. Surgery 1996;119:498-504.

32. Uesaka K, Nimura Y, Nagino M. Changes in hepatic lobar function after right portal vein embolization. An appraisal by biliary indocyanine green excretion. Ann Surg 1996;223:77-83.

33. Kaiho T, Miyazaki M, Ito H, Ambiru S, Shimizu H, Togawa A, et al. Reduced hepatic functional reserve in cirrhosis and obstructive jaundice with special reference to histological morphometric analysis and galactose elimination capacity. Eur Surg Res 1996;28:333-40.

34. Kwon AH, Ha-Kawa SK, Uetsuji S, Kamiyama Y, Tanaka Y. Use of technetium $99 \mathrm{~m}$ diethylenetriamine-pentaacetic acidgalactosyl-human serum albumin liver scintigraphy in the evaluation of preoperative and postoperative hepatic functional reserve for hepatectomy. Surgery 1995;117:429-34.

35. Kayahara M, Nagakawa T, Ohta T, Kitagawa H, Tajima H, Miwa $\mathrm{K}$. Role of nodal involvement and the periductal soft-tissue margin in middle and distal bile duct cancer. Ann Surg 1999;229:76-83.

36. Miyazaki M, Ito H, Nakagawa K, Ambiru S, Shimizu H, Shimizu Y, et al. Aggressive surgical approaches to hilar cholangiocarcinoma: hepatic or local resection? Surgery 1998;123:131-6.

37. Jang JY, Kim SW, Park DJ, Ahn YJ, Yoon YS, Choi MG, et al. Actual long-term outcome of extrahepatic bile duct cancer after surgical resection. Ann Surg 2005;241:77-84.

38. Ikeyama T, Nagino M, Oda K, Ebata T, Nishio H, Nimura Y. Surgical approach to Bismuth type I and II hilar cholangiocarcinomas: audit of 54 consecutive cases. Ann Surg 2007;246: 1052-7.

39. Mizumoto R, Kawarada Y, Suzuki H. Surgical treatment of hilar carcinoma of the bile duct. Surg Gynecol Obstet 1986;162: $153-8$.

40. Nimura Y, Hayakawa N, Kamiya J, Kondo S, Shionoya S. Hepatic segmentectomy with caudate lobe resection for bile duct carcinoma of the hepatic hilus. World J Surg 1990;14:535-43.

41. Kamiya J, Nimura Y, Hayakawa N, Kondo S, Nagino M, Kanai $\mathrm{M}$. Preoperative cholangiography of the caudate lobe: surgical anatomy and staging for biliary carcinoma. J Hepatobiliary Pancreat Surg 1994;4:385-9.

42. Nimura Y, Hayakawa N, Kamiya J, Kondo S, Nagino M, Kanai M. Hilar cholangiocarcinoma: surgical anatomy and curative resection. J Hepatobiliary Pancreat Surg 1995;2:239-48.

43. Su CH, Tsay SH, Wu CC, Shyr YM, King KL, Lee CH, et al. Factors influencing postoperative morbidity, mortality, and survival after resection for hilar cholangiocarcinoma. Ann Surg 1996;223:384-94.

44. Chamberlain RS, Blumgart LH. Hilar cholangiocarcinoma: a review and commentary. Ann Surg Oncol 2000;7:55-66.
45. Boerma EJ. Research into the results of resection of hilar bile duct cancer. Surgery 1990;108:572-80.

46. Kuvshinoff BW, Fong Y, Blumgart LH. Proximal bile duct tumors. Surg Oncol Clin N Am 1996;5:317-36.

47. Gazzaniga GM, Filauro M, Bagarolo C, Mori L. Surgery for hilar cholangiocarcinoma: Italian experience. J Hepatobiliary Pancreat Surg 2000;7:122-7.

48. Lee SG, Lee YJ, Park KM, Hwang S, Min PC. One hundred and eleven liver resections for hilar bile duct cancer. J Hepatobiliary Pancreat Surg 2000;7:135-41.

49. Ebata T, Nagino M, Kamiya J, Uesaka K, Nagasaka T, Nimura Y. Hepatectomy with portal vein resection for hilar cholangiocarcinoma: audit of 52 consecutive cases. Ann Surg 2003;238: $720-7$.

50. Miyazaki M, Kato A, Ito H, Kimura F, Shimizu H, Ohtsuka M, et al. Combined vascular resection in operative resection for hilar cholangioma: does it work or not? Surgery 2007;141: $581-8$.

51. Suzuki T, Yoshidome H, Kimura F, Shimizu H, Ohtsuka M, Kato A, et al. Renal function is well maintained after use of left renal vein graft for vascular reconstruction in hepatobiliarypancreatic surgery. J Am Coll Surg 2006;202:87-92.

52. Kondo S, Katoh H, Hirano S, Ambo Y, Tanaka E, Okushiba S. Portal vein resection and reconstruction prior to hepatic dissection during right hepatectomy and caudate lobectomy for hepatobiliary cancer. Br J Surg 2003;90:694-7.

53. Hemming AW, Kim RD, Mekeel KL, Fujita S, Reed AI, Foley $\mathrm{DP}$, et al. Portal vein resection for hilar cholangiocarcinoma. Am Surg 2006;72:599-604.

54. Neuhaus P, Jonas S, Bechstein WO, Lohmann R, Radke C, Kling $\mathrm{N}$, et al. Extended resections for hilar cholangiocarcinoma. Ann Surg 1999;230:808-18.

55. Sugiura Y, Nakamura S, Iida S, Hosoda Y, Ikeuchi S, Mori S, et al. Extensive resection of the bile ducts combined with liver resection for cancer of the main hepatic duct junction: a cooperative study of the Keio Bile Duct Cancer Study Group. Surgery 1994;115:445-51.

56. Nimura Y, Kamiya J, Kondo S, Nagino M, Uesaka K, Oda K, et al. Aggressive preoperative management and extended surgery for hilar cholangiocarcinoma: Nagoya experience. J Hepatobiliary Pancreat Surg 2000;7:155-62.

57. Klempnauer J, Ridder GJ, Werner M, Weimann A, Pichlmayr R. What constitutes long-term survival after surgery for hilar cholangiocarcinoma? Cancer 1997;79:26-34.

58. Pichlmayr R, Weimann A, Klempnauer J, Oldhafer KJ, Maschek $\mathrm{H}$, Tusch $\mathrm{G}$, et al. Surgical treatment in proximal bile duct cancer: a single-center experience. Ann Surg 1996;224:628-38.

59. Kosuge T, Yamamoto J, Shimada K, Yamasaki S, Makuuchi M. Improved surgical results for hilar cholangiocarcinoma with procedures including major hepatic resection. Ann Surg 1999; 230:663-71.

60. Wakai T, Shirai Y, Moroda T, Yokoyama N, Hatakeyama K. Impact of ductal resection margin status on long-term survival in patients undergoing resection for extrahepatic cholangiocarcinoma. Cancer 2005;103:1210-6.

61. Bhuiya MR, Nimura Y, Kamiya J, Kondo S, Fukata S, Hayakawa N, et al. Clinicopathological studies on perineural invasion of bile duct cancer. Ann Surg 1992;215:344-9.

62. Miyazaki M, Ito H, Nakagawa K, Ambiru S, Shimizu H, Okaya $\mathrm{T}$, et al. Parenchyma-preserving hepatectomy in the surgical treatment of hilar cholangiocarcinoma. J Am Coll Surg 1999;189:575-83.

63. Sakamoto $\mathrm{Y}$, Kosuge T, Shimada K, Sano T, Ojima H, Yamamoto J, et al. Prognostic factors of surgical resection in middle and distal bile duct cancer: an analysis of 55 patients concerning the significance of ductal and radial margins. Surgery 2005;137:396-402.

64. Yeh CN, Jan YY, Chao TC, Chen MF. Laparoscopic cholecystectomy for polypoid lesions of the gallbladder: a clinicopatho- 
logic study. Surg Laparosc Endosc Percutan Tech 2001;11: 176-81.

65. Wakai T, Shirai Y, Yokoyama N, Nagakura S, Watanabe H, Hatakeyama K. Early gallbladder carcinoma does not warrant radical resection. Br J Surg 2001;88:675-8.

66. Ouchi K, Mikuni J, Kakugawa Y; Organizing Committee, The 30th Annual Congress of the Japanese Society of Biliary Surgery. Laparoscopic cholecystectomy for gallbladder carcinoma: results of a Japanese survey of 498 patients. J Hepatobiliary Pancreat Surg 2002;9:256-60.

67. Wibbenmeyer LA, Wade TP, Chen RC, Meyer RC, Turgeon RP, Andrus $\mathrm{CH}$. Laparoscopic cholecystectomy can disseminate in situ carcinoma of the gallbladder. J Am Coll Surg 1995;181: 504-10.

68. Sano T, Ajiki T, Hirata K, Okazaki T, Fujino Y, Suzuki Y, et al. A recurrent case of an early gallbladder carcinoma after laparoscopic cholecystectomy. Hepatogastroenterology 2004;51:672-4.

69. Paolucci V, Schaeff B, Schneider M, Gutt C. Tumor seeding following laparoscopy: international survey. World J Surg 1999;23:989-95.

70. Lundberg O, Kristoffersson A. Port site metastases from gallbladder cancer after laparoscopic cholecystectomy: results of a Swedish survey and review of published reports. Eur J Surg 1999;165:215-22.

71. Z'graggen K, Birrer S, Maurer CA, Wehrli H, Klaiber C, Baer HU. Incidence of port site recurrence after laparoscopic cholecystectomy for preoperatively unsuspected gallbladder carcinoma. Surgery 1998;124:831-8.

72. Lundberg O, Kristoffersson A. Open versus laparoscopic cholecystectomy for gallbladder carcinoma. J Hepatobiliary Pancreat Surg 2001;8:525-9.

73. Whalen GF, Bird I, Tanski W, Russell JC, Clive J. Laparoscopic cholecystectomy does not demonstrably decrease survival of patients with serendipitously treated gallbladder cancer. J Am Coll Surg 2001;192:189-95.

74. Kokudo N, Makuuchi M, Natori T, Sakamoto Y, Yamamoto J, Seki M, et al. Strategies for surgical treatment of gallbladder carcinoma based on information available before resection. Arch Surg 2003;138:741-50.

75. Varshney S, Buttirini G, Gupta R. Incidental carcinoma of the gallbladder. Eur J Surg Oncol 2002;28:4-10.

76. Yamaguchi K, Chijiiwa K, Ichimiya H, Sada M, Kawakami K, Nishikata F, et al. Gallbladder carcinoma in the era of laparoscopic cholecystectomy. Arch Surg 1996;131:981-4.

77. Shirai Y, Yoshida K, Tsukada K, Muto T. Inapparent carcinoma of the gallbladder: an appraisal of a radical second operation after simple cholecystectomy. Ann Surg 1992;215: 326-31.

78. Box JC, Edge SB. Laparoscopic cholecystectomy and unsuspected gallbladder carcinoma. Semin Surg Oncol 1999;16: 327-31.

79. Wakai T, Shirai Y, Yokoyama N, Ajioka Y, Watanabe H, Hatakeyama K. Depth of subserosal invasion predicts long-term survival after resection in patients with T2 gallbladder carcinoma. Ann Surg Oncol 2003;10:447-54.

80. Suzuki S, Yokoi Y, Kurachi K, Inaba K, Ota S, Azuma M, et al. Appraisal of surgical treatment for pT2 gallbladder carcinomas. World J Surg 2004;28:160-5.

81. Shimizu Y, Ohtsuka M, Ito H, Kimura F, Shimizu H, Togawa A, et al. Should the extrahepatic bile duct be resected for locally advanced gallbladder cancer? Surgery 2004;136:1012-7.

82. Tsukada K, Hatakeyama K, Kurosaki I, Uchida K, Shirai Y, Muto T, et al. Outcome of radical surgery for carcinoma of the gallbladder according to the TNM stage. Surgery 1996;120: 816-21.

83. Shimada H, Endo I, Fujii Y, Kamiya N, Masunari H, Kunihiro O, et al. Appraisal of surgical resection of gallbladder cancer with special reference to lymph node dissection. Langenbecks Arch Surg 2000;385:509-14.
84. Chijiiwa K, Nakano K, Ueda J, Noshiro H, Nagai E, Yamaguchi $\mathrm{K}$, et al. Surgical treatment of patients with T2 gallbladder carcinoma invading the subserosal layer. J Am Coll Surg 2001; 192:600-7.

85. Schauer RJ, Meyer G, Baretton G, Schildberg FW, Rau HG. Prognostic factors and long-term results after surgery for gallbladder carcinoma: a retrospective study of 127 patients. Langenbecks Arch Surg 2001;386:110-7.

86. de Aretxabala X, Roa I, Burgos L, Losada H, Roa JC, Mora J, et al. Gallbladder cancer: an analysis of a series of 139 patients with invasion restricted to the subserosal layer. J Gastrointest Surg 2006;10:186-92.

87. Matsusaka S, Yamasaki H, Kitayama Y, Okada T, Maeda S. Occult gallbladder carcinoma diagnosed by a laparoscopic cholecystectomy. Surg Today 2003;33:740-2.

88. Weiland ST, Mahvi DM, Niederhuber JE, Heisey DM, Chicks DS, Rikkers LF. Should suspected early gallbladder cancer be treated laparoscopically? J Gastrointest Surg 2002;6:50-6.

89. Mori T, Souda S, Hashimoto J, Yoshikawa Y, Ohshima M. Unsuspected gallbladder cancer diagnosed by laparoscopic cholecystectomy: a clinicopathological study. Surg Today 1997;27: 710-3.

90. de Aretxabala X, Roa I, Burgos L. Gallbladder cancer: management of early tumors. Hepatogastroenterology 1999;46:154751.

91. Ohtsuka M, Miyazaki M, Itoh H, Nakagawa K, Ambiru S, Shimizu H, et al. Routes of hepatic metastasis of gallbladder carcinoma. Am J Clin Pathol 1998;109:62-8.

92. Bartlett DL, Fong Y, Fortner JG, Brennan MF, Blumgart LH. Long-term results after resection for gallbladder cancer: implications for staging and management. Ann Surg 1996;224:63946.

93. Onoyama H, Yamamoto M, Tseng A, Ajiki T, Saitoh Y. Extended cholecystectomy for carcinoma of the gallbladder. World J Surg 1995;19:758-63.

94. Kondo S, Nimura Y, Hayakawa N, Kamiya J, Nagino M, Uesaka $\mathrm{K}$. Extensive surgery for carcinoma of the gallbladder. Br J Surg 2002;89:179-84.

95. Yamaguchi R, Nagino M, Oda K, Kamiya J, Uesaka K, Nimura Y. Perineural invasion has a negative impact on survival of patients with gallbladder carcinoma. Br J Surg 2002;89:1130-6.

96. Kondo S, Nimura Y, Kamiya J, Nagino M, Kanai M, Uesaka K, et al. Factors influencing postoperative hospital mortality and long-term survival after radical resection for stage IV gallbladder carcinoma. World J Surg 2003;27:272-7.

97. Sasaki E, Nagino M, Ebata T, Oda K, Arai T, Nishio H, et al. Immunohistochemically demonstrated lymph node micrometastasis and prognosis in patients with gallbladder carcinoma. Ann Surg 2006;244:99-105.

98. Tsukada K, Kurosaki I, Uchida K, Shirai Y, Oohashi Y, Yokoyama N, et al. Lymph node spread from carcinoma of the gallbladder. Cancer 1997;80:661-7.

99. Miyazaki M, Itoh H, Ambiru S, Shimizu H, Togawa A, Gohchi E, et al. Radical surgery for advanced gallbladder carcinoma. Br J Surg 1996;83:478-81.

100. Shirai Y, Ohtani T, Tsukada K, Hatakeyama K. Combined pancreaticoduodenectomy and hepatectomy for patients with locally advanced gallbladder carcinoma: long term results. Cancer 1997;80:1904-9.

101. Kondo S, Nimura Y, Kamiya J, Nagino M, Kanai M, Uesaka K, et al. Five-year survivors after aggressive surgery for stage IV gallbladder cancer. J Hepatobiliary Pancreat Surg 2001;8: 511-7.

102. Yamaguchi K, Enjoji M. Carcinoma of the ampulla of vater: a clinicopathologic study and pathologic staging of 109 cases of carcinoma and 5 cases of adenoma. Cancer 1987;59:50615.

103. Beger HG, Thorab FC, Liu Z, Harada N, Rau BM. Pathogenesis and treatment of neoplastic diseases of the papilla of Vater: 
Kausch-Whipple procedure with lymph node dissection in cancer of the papilla of Vater. J Hepatobiliary Pancreat Surg 2004; 11:232-8.

104. Knox RA, Kingston RD. Carcinoma of the ampulla of Vater. Br J Surg 1986;72:72-3.

105. Goldberg M, Zamir O, Hadary A, Nissan S. Wide local excision as an alternative treatment for periampullary carcinoma. Am J Gastroenterol 1987;82:1168-71.

106. Sharp KW, Barandes JL. Local resection of tumor of the ampulla of Vater. Am Surg 1990;56:214-7.

107. Branum GD, Pappas TN, Meyers WC. The management of tumors of the ampulla of Vater by local resection. Ann Surg 1996;224:621-7.

108. Klein P, Reingruber B, Kastl S, Dworak O, Hohenberger W. Is local excision of pT1-ampullary carcinomas justified? Eur J Surg Oncol 1996;22:366-71.

109. Paramythiotis D, Kleeff J, Wirtz M, Friess H, Buchler MW. Still any role for transduodenal local excision in tumors of the papilla of Vater? J Hepatobiliary Pancreat Surg 2004;11:239_ 44.

110. Beger HG, Treitschke F, Gansauge F, Harada N, Hiki N, Mattfeldt T. Tumor of the ampulla of Vater. Arch Surg 1999;134: 526-32.
111. Kayahara M,Nagakawa T,Ueno K, Ohta T, Tsukioka Y, Miyazaki I. Surgical strategy for carcinoma of the pancreas head area based on clinicopathologic analysis of nodal involvement and plexus invasion. Surgery 1995;117:616-23.

112. Shirai Y, Ohtani T, Tsukada K, Hatakeyama K. Patterns of lymphatic spread of carcinoma of the ampulla of Vater. Br J Surg.1997;84:1012-6.

113. Kayahara M, Nagakawa T, Ohta T, Kitagawa H, Miyazaki I. Surgical strategy for carcinoma of the papilla of Vater based on the lymphatic flow and mode of recurrence. Surgery 1997;121: 611-7.

114. Mizuno T, Ishizaki Y, Ogura K, Yoshimoto J, Kawasaki S. Clinical significance of immunohistochemically detectable lymph node metastasis in adenocarcinoma of the ampulla of Vater. Br J Surg 2006;93:221-5.

115. Chan C, Herrera MF, de la Garza L, Quintanilla-Martinez L, Vargas-Vorackova F, Richaud-Patin Y, et al. Clinical behavior and prognsostic factors of periampullary adenocarcinoma. Ann Surg 1995;222:632-7. 